\title{
Formación en coaching educativo: aprendizajes y retos
}

\section{Educational coaching programme: learnings and challenges ahead}

DOI: $10.46932 / \mathrm{sfjdv} 2 \mathrm{n} 2-122$

Received in: March 1st, 2021

Accepted in: May 30th, 2021

\author{
Jhon Baquero Baratoa \\ Departamento de Psicología, Universidad Autónoma de Madrid, Madrid, España \\ Ciudad Universitaria de Cantoblanco $\cdot 28049$ Madrid, España \\ E-mail: alexander.baquero@gmail.com \\ María Rodríguez Moneob \\ Departamento de Psicología, Universidad Autónoma de Madrid, Madrid, España \\ Teresa Cardona de Cortésc \\ Departamento de Psicología, Universidad de los Andes, Bogotá, Colombia
}

\section{RESUMEN}

El coaching, como metodología para gestionar exitosamente el desarrollo de las personas y el cambio en las organizaciones, ha trascendido desde las empresas hacia su uso en todo el proceso educativo, incluyendo las prácticas docentes que ocurren en el aula. Es así como hace poco más de una década se empezó a consolidar el Coaching Educativo dentro de instituciones del Reino Unido, Estados Unidos y Australia. Este trabajo presenta los resultados del análisis de entrevistas y testimonios escritos de veintidós profesores participantes de un programa de formación en Coaching Educativo implementado en la Universidad de los Andes (Bogotá - Colombia), acerca de sus motivaciones para participar en el programa, los aprendizajes obtenidos y los retos que han encontrado en su aplicación. En general, las conclusiones muestran que el programa incrementó la conciencia de los profesores participantes acerca de su rol en el proceso de enseñanza-aprendizaje, su concepción acerca de los estudiantes, así como del proceso educativo mismo. También se hace referencia a las dificultades encontradas en la aplicación de las herramientas y aprendizajes obtenidos durante el programa, que son aspectos que deben ser considerados por las intervenciones orientadas a la transformación del rol docente.

Palabras clave: Coaching; Coaching educativo; Rol docente; Transformación docente

\begin{abstract}
Coaching, as a methodology to successfully manage people's development and change in organizations, has gone from being used in companies to be used throughout the educational process, even as teaching practices that occur in the classroom. Coaching in Education began to consolidate itself within the institutions of the United Kingdom, the United States and Australia little more than a decade ago. This paper presents the analysis of the interviews and written testimonials of twenty two (22) participants in a training program in Coaching in Education which has been developed and implemented at the University of the Andes (Bogotá - Colombia), about their motivations to participate in the program, their learnings and the challenges they have encountered in its application. In general, the conclusions show that the program increases the awareness of the teachers who participate in the learning process, widened their conception about the students, as well as the educational process itself. It also refers to the difficulties encountered in the application of tools and learnings, which are aspects that must be addressed by interventions aimed at the transformation of teachers.
\end{abstract}


Keywords: Coaching; Educational coaching; Teaching role; Teacher transformation

\section{INTRODUCCIÓN}

Dada la importancia que tiene la educación en la sociedad como movilizadora de la humanidad, ésta debe potencializar el desarrollo de habilidades que permitan la construcción de conocimiento nuevo, la apropiación de valores, el desarrollo de competencias, la formación del autoconcepto, y el establecimiento y concreción de metas (Sánchez-Teruel, 2013). Por esta razón es crucial que las instituciones de educación, y particularmente los profesores, implementen nuevos modelos educativos y pedagógicos, que promuevan la transformación del rol docente, y que den mayor relevancia al aprendizaje del estudiante, a fomentar su autoconocimiento y adquisición de posturas críticas y creativas, alineadas con el aprovechamiento de los avances tecnológicos, con el desarrollo de la sociedad y con las demandas de la Sociedad del Siglo XXI (Pascual \& Leonor, 2016).

Todos estos elementos confluyen en la oportunidad de emplear estrategias, habilidades y herramientas que provienen del coaching y que están siendo aplicadas en entornos educativos de países como Inglaterra, Australia y Estados Unidos. El coaching es una práctica que facilita la identificación del potencial de los individuos, fomenta su autoconocimiento y sentido de responsabilidad, estimula su capacidad para aprender a aprender, y mejora su rendimiento (Whitmore, 2003). Este trabajo describe un proyecto de formación en coaching educativo para profesores implementado en el año 2018 en la Universidad de los Andes (Bogotá, Colombia) y los resultados obtenidos en términos de los aprendizajes logrados por algunos de los participantes y de su implementación en su práctica educativa específica.

\section{MARCO TEÓRICO}

El coaching como metodología para gestionar exitosamente el desarrollo de las personas y el cambio, ha trascendido desde las empresas hacia su uso en todo el proceso educativo, incluyendo las prácticas que ocurren en el aula. Su aplicación promueve, entre otras, la autonomía de los estudiantes, el autoaprendizaje y la capacidad de aprender a aprender, y el desarrollo de competencias (Obiols \& Giner, 2011; Sánchez \& Boronat, 2014; Van Nieuwerburgh, 2012).

Pese a que existen múltiples definiciones para coaching, Rosinski (2008) afirma que es principalmente un proceso conversacional, diseñado para facilitar el logro de objetivos, a través de la implementación de actividades y estrategias que ayudan al individuo a identificar sus fortalezas, anticipar obstáculos, desarrollar habilidades y usar apropiadamente los recursos disponibles. En otras palabras, el coaching puede comprenderse como un proceso de acompañamiento que ocurre a través de conversaciones estructuradas, en las que se ayuda a las personas a definir sus metas, explorar sus 
creencias, desarrollar hábitos, definir planes de acción y monitorear su implementación para fomentar el logro de los resultados (Bou, 2007; Rogers, 2012; Villa, Caperán \& Caperán, 2010; Hall \& Duval, 2010).

La esencia de los procesos de coaching está constituida por cuatro elementos: a) la adquisición de conciencia y aceptación personal; b) el abandono de la zona de confort y la delimitación de metas motivadoras; c) el avance gradual y constante, siguiendo la intuición y manejando el tiempo; y d) la reflexión permanente (Carril, 2008). Algunos trabajos de reflexión teórica e investigación, han planteado que el coaching puede promover el desarrollo de la autorregulación (Baquero Barato \& Rodríguez Moneo, 2016, 2021), poniendo de manifiesto algunas ventajas que su aplicación en entornos educativos podría aportar para el desarrollo y la formación integral de los estudiantes.

Respecto al rol docente, el coaching se destaca como una estrategia que promueve la función colaborativa de los profesores. También facilita la transformación del rol docente desde la transmisión de información, hacia el acompañamiento del proceso de aprendizaje de los estudiantes, movilizando sus creencias y sus emociones (Anderson, 2010; Gornall \& Burn, 2013; Mitchell \& Gansemer-Topf, 2016; Rhodes \& Fletcher, 2012; Rose, McGuire-Snieckus \& Gilbert, 2015). Es decir que, en línea con lo anterior, los profesores podrían desarrollar las habilidades propias del coaching para implementar conversaciones con sus estudiantes que promuevan su desarrollo integral, trascendiendo el aprendizaje exclusivamente de conceptos, y abordando otras temáticas y habilidades que son de interés para los alumnos en su preparación para los retos que la sociedad actual plantea.

\section{PLANTEAMIENTO DEL PROBLEMA}

Para implementar el coaching, los docentes requieren de un entrenamiento que les permita adquirir las habilidades requeridas para construir entornos basados en la confianza, la empatía y la colaboración con sus estudiantes. De igual manera, los profesores deben prepararse para implementar metodologías que faciliten el logro de objetivos, la toma de conciencia, la toma de decisiones, el sentido de responsabilidad y la autonomía de sus alumnos (Whitmore, 2003; Hoekstra \& Korthagen, 2011).

En diversos estudios (ver Cortés, 2012), se ha planteado que el desarrollo docente y la implementación efectiva de estos cambios, requieren de la implicación institucional y personal de parte de los profesores, con el objetivo de hacer una revisión acerca de los roles, las responsabilidades, las habilidades y los recursos requeridos en esta nueva función docente. También se deben revisar los esquemas de soporte metodológico, y las resistencias que pueden surgir durante el proceso de transformación y que pueden limitar su adopción. El presente trabajo explora los aprendizajes obtenidos a partir de la participación de un grupo de docentes de diferentes niveles educativos, un programa de 
formación en coaching educativo, los impactos percibidos y las dificultades que han tenido en su aplicación.

\section{MÉTODO}

\subsection{PARTICIPANTES}

Veintidós (22) profesores de diferentes niveles educativos, participaron del programa Coaching Educativo realizado en la Universidad de los Andes (Bogotá) en el año 2018, como parte de la oferta de Educación Continuada que brinda la Universidad. Específicamente, trece (13) eran profesores de colegio, tres (3) ocupaban cargos directivos en colegios, cuatro (4) se desempeñaban como consejeros académicos y dos (2) eran profesores universitarios.

\subsection{PROCEDIMIENTO}

Se realizó una entrevista semiestructurada a doce (12) de los participantes, cuya duración aproximada fue de 1 hora, después de un mes de haber finalizado el curso. Por dificultades de agenda, los 10 participantes restantes enviaron un testimonio escrito que abordaba los mismos temas cubiertos en la entrevista. En ambos casos (entrevista y testimonio escrito), se indagó acerca del nivel de satisfacción con relación al programa, a los aprendizajes obtenidos, del uso de estos aprendizajes en su práctica cotidiana y a las dificultades encontradas para su aplicación.

\subsection{PROGRAMA DE COACHING EDUCATIVO}

El curso Coaching Educativo ha hecho parte de la oferta de cursos de Educación Continuada de la Universidad de los Andes por varios años. Es un programa presencial, de 32 horas de duración, cuyo objetivo es promover que los participantes adquieran una concepción integral de sí mismos y de sus estudiantes, desarrollen habilidades comunicacionales, conozcan modelos y herramientas propias del coaching, y reflexionen acerca del rol docente como facilitador del aprendizaje.

Específicamente está compuesto de seis módulos: 1. Introducción al coaching; 2. Proceso y modelos de coaching educativo; 3. Habilidades de coaching; 4. Habilidades de comunicación; 5. Emociones y valores; 6. Aprendizajes y plan de acción individual. La gráfica 1 presenta los temas más relevantes abordados durante cada módulo. 


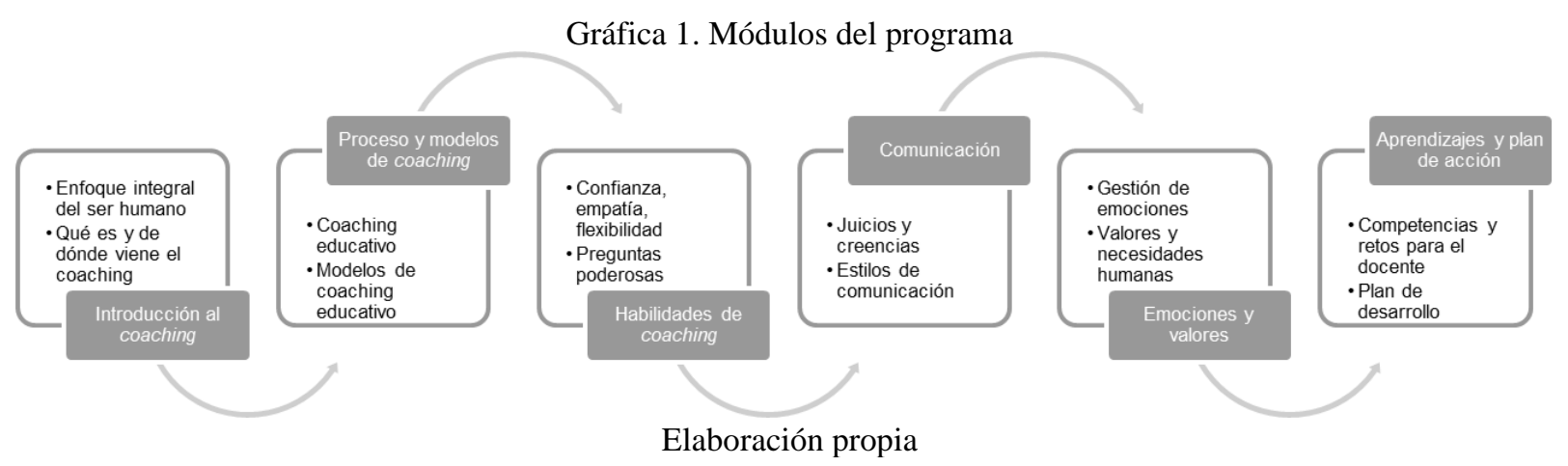

\section{RESULTADOS}

A partir de la estructura de las entrevistas y de los testimonios escritos, los resultados se analizaron en tres bloques: los aprendizajes obtenidos, el impacto percibido (en el proceso de enseñanza, en la concepción del rol del profesor, en la relación profesor - estudiante y en el rendimiento académico) y, por último, las dificultades encontradas.

\subsection{APRENDIZAJES OBTENIDOS}

Los participantes resaltaron haber aprendido a conocerse a sí mismos, a hacer introspección: "si tú eres autoconsciente de tus prácticas, tú puedes primero transformarte y luego transformar al otro con una comunicación que realmente fluya".

Algunos participantes mencionaron que, antes del curso, no veían la importancia de conocer a sus estudiantes: "llegar con los ojos vendados a dictar la clase, sin importarme finalmente lo que ellos son", y mencionan la transformación que hubo después del programa: “aprender a conocerlos y a manejar grupos; yo antes, cuando no era tan consiente de estas cosas, decía, pero porqué esa estrategia me funciona con unos, pero no con otros".

Otro aprendizaje se vincula con la capacidad de hacer preguntas para mejorar la interacción con padres de familia y demás adultos del ambiente escolar, pero también para promover que los estudiantes sean activos y autónomos en su proceso de aprendizaje: "entendimos el poder de la pregunta; eso modifico la forma de cuestionar a los muchachos para que ellos, al responder, aprendan”. Esto facilita que los estudiantes compartan sus propios objetivos: "qué es lo que quieres, qué es lo principal, entonces eso me ha servido bastante para guiarlos".

Por último, conocer los estilos de comunicación impactó el interés por observar comportamientos, comprender las emociones y hacer introspección periódica: "me marco el tema de la comunicación; poder verme a mí mismo, cómo me comunico con los demás y cómo las otras personas se comunican de manera diferente. Ver que uno a veces no reconoce eso y al ser consciente de ello, puedes obtener mejores resultados". 


\subsection{IMPACTO EN EL PROCESO DE ENSEÑANZA}

En cuanto a la manera de enseñar, se cuestionaron las metodologías que utilizaban. Los participantes terminaron más motivados a crear ambientes prácticos y participativos: "cambió bastante porque nosotros, por la rutina, dejamos de cautivar y por el cansancio, por lo que sea dejamos de motivar, dejamos de lado ciertas prácticas que son muy buenas y que nos facilitan las cosas", "preguntarles cosas, de hacer la clase mucho más de ellos, hablando, haciendo y construyendo, y no solamente yo dando"

Los participantes también se cuestionaron sobre los métodos de evaluación: “¿Qué necesitamos saber cuando evaluamos esas competencias? ¿cómo las evaluamos?”. Ahora, más que memorización, se busca que el estudiante pueda relacionar los contenidos de clase con sus experiencias: "aplicar e ir más allá; tratar de sacar cosas para la vida en general, y no solamente para el examen".

\subsection{IMPACTO EN LA CONCEPCIÓN DEL ROL DEL PROFESOR}

Se incrementó la consciencia acerca de la importancia de permitir que estudiante aporte en los objetivos y construya la clase: "que no solo haga lo que yo lo que yo necesito que hagan, sino que construyan lo que ellos también esperan hacer".

Se incrementó el compromiso de los profesores con el desarrollo personal de los estudiantes. Más que inducir al alumno a cumplir con los ideales de los profesores, se formulan preguntas para que el estudiante encuentre sus propias respuestas: "Tengo conversaciones diferentes con mis estudiantes, les pregunto qué pasa, por qué reaccionan como reaccionan, y no hay necesidad de hacer anotaciones, ni llamar a los padres; seguramente eso es una experiencia de vida para el estudiante".

\subsection{IMPACTO EN LA RELACIÓN ENTRE EL DOCENTE Y EL ESTUDIANTE}

Reconocer la individualidad de cada estudiante permite construir relaciones cercanas entre los profesores y el grupo, lo que aporta tranquilidad para estudiantes y docentes. Dentro y fuera del salón se percibe una relación más armónica, dinámica, y construida por todos y para todos: "empezar con dinámicas suaviza mucho y le da a uno mucha tranquilidad también; poder hablar y poder realizar cosas diferentes para poder concentrar a la gente en lo que uno quiere", "las niñas quedan tranquilas, como que llegan con preguntas y quieren encontrar sus respuestas de vida que son tan importantes".

En general, los estudiantes se muestran más comprometidos con el desarrollo de la clase y con el cumplimiento de sus deberes, están contentos, motivados, atentos y afectuosos durante las sesiones: "los estudiantes se sienten, se apersonan tanto que hasta motivan a sus compañeros”. 


\subsection{IMPACTO EN LOS RESULTADOS ACADÉMICOS DE LOS ESTUDIANTES}

Aunque el impacto no es tangible aún, los participantes comentan que han percibido algunas mejorías en el rendimiento académico de los estudiantes. Sienten que, al plantearles preguntas, los estudiantes realizan un proceso de reflexión mucho más valioso que el simple almacenamiento de información. Durante las entrevistas, una de las participantes compartió su experiencia con un estudiante, quien le afirmó "no vas a esperar que yo saque la mejor nota en tu materia, pero si he aprendido muchas cosas".

\subsection{DIFICULTADES PERCIBIDAS PARA LA APLICACIÓN DE LOS APRENDIZAJES}

Los participantes señalan que, muchas veces, olvidan las herramientas a la hora de planear las clases: "no tenemos eso metido en la programación". Esto genera que se retorne a la metodología tradicional, y que se controlen y evalúen competencias distintas: "uno se preocupa mucho por la disciplina, entonces controla la disciplina; se olvida lo que queremos de la clase, qué queremos que aprendan".

Se identifica, además, el tiempo como una gran dificultad; por un lado, en el afán de la cotidianidad, los docentes no tienen la oportunidad de planear las clases con la metodología aprendida. Por otro lado, la duración de las sesiones es corta para aplicar todas las herramientas.

Otra dificultad se relaciona con la cantidad de estudiantes en cada grupo. Es complicado establecer un diálogo con cada estudiante, así que la aproximación debe ser mucho más superficial. Además, un profesor comenta la dificultad que tiene para establecer objetivos con grupos numerosos, ya que "las metas son diferentes para todos los chicos".

\section{DISCUSIÓN}

Los comentarios de los participantes, a través de sus testimonios escritos y de las entrevistas realizadas, fueron analizados en tres bloques principales: los aprendizajes obtenidos, el impacto percibido y las dificultades encontradas en el momento de aplicar el coaching educativo en los entornos académicos.

Con relación al primer punto, los participantes comentan haber obtenido aprendizajes vinculados con la capacidad de hacer introspección, con la importancia de conocer a sus estudiantes, e implementar herramientas como preguntar e identificar los estilos de comunicación para fomentar la participación de todos. Estos aprendizajes han impactado de manera positiva aspectos como el proceso de enseñanza, la concepción del rol del profesor, y la concepción de los estudiantes, en donde los participantes afirman haber cambiado las prácticas tradicionales, y estar utilizando estas herramientas para involucrar más a los estudiantes en su proceso formativo. Estos aprendizajes están alineados con las competencias que se 
esperan de los docentes en los nuevos paradigmas educativos como, por ejemplo, en la educación por competencias (Obiols \& Giner, 2011).

De esta manera, los participantes afirman haber iniciado el proceso de transformación del rol docente, migrando desde la mera transmisión de información hasta acompañar el proceso del estudiante, empoderándolo e involucrándolo permanentemente. El acompañamiento integral al proceso de aprendizaje del estudiante, abarca aspectos como la gestión emocional, la gestión de las creencias y el involucramiento activo en el logro de metas (Anderson, 2010; Gornall \& Burn, 2013). Esta transformación está impactando positivamente el relacionamiento entre profesores y estudiantes, así como el desempeño académico. El impacto en el desempeño académico podría ser un indicador de la posible relación entre el uso del coaching y el desarrollo de la autorregulación (Baquero Barato \& Rodríguez Moneo, 2016, 2021) y de la autoeficacia (Rhodes \& Fletcher, 2012).

Pese a estos resultados positivos, sin embargo, los participantes mencionan dificultades que han tenido al momento de llevar a la práctica estos aprendizajes. Algunos de estos inconvenientes están relacionados con aspectos institucionales y administrativos del rol docente, como lo son el tiempo de las clases, el tiempo de preparación y el tamaño de los grupos. También indican la necesidad de construir redes de apoyo dentro de las instituciones educativas que permitan acompañarse entre sí en la implementación del coaching educativo. Estos desafíos identificados por los docentes, están en línea de lo que plantean autores como Cortés (2012) en relación con la importancia de la implicación institucional y personal, para poder llevar a cabo los cambios y transformaciones requeridas.

Otras dificultades están relacionadas con tener presente los cambios y los aprendizajes al momento de preparar las sesiones, y que pueden indicar la necesidad de tener acompañamientos y sesiones de refuerzo posteriores a la formación, para acompañar a los docentes en este proceso de transición hacia un nuevo paradigma educativo.

Como dirección de futuras investigaciones, es importante considerar el impacto que tiene esta formación en variables como el clima de aula y el desempeño académico, empleando estrategias de medición directa, dado que este trabajo se basó únicamente en las percepciones y consideraciones de los profesores que participaron en el curso.

\section{CONCLUSIONES}

Este trabajo describe la experiencia del curso Coaching Educativo ofrecido por Educación Continua de la Universidad de los Andes, en Bogotá, a partir de entrevistas realizadas y testimonios escritos obtenidos de veintidós (22) participantes, y se enfoca específicamente en los aprendizajes obtenidos durante la formación, el impacto generado en aspectos como la concepción del rol docente y la 
relación entre estudiantes y profesores, y en las dificultades encontradas para aplicarlos en la entornos reales.

El coaching se está utilizando cada vez más en entornos educativos de diversos países. Su aplicación demanda que los profesores reciban la formación requerida, desarrollen habilidades específicas y adquieran herramientas para ponerlo en práctica. A partir de este trabajo, se concluye que esta formación generó aprendizajes de diferente naturaleza, desde la necesidad de hacer introspección, cuestionar el propio rol, hasta la efectividad que hacer preguntas y conocer los estilos de comunicación.

De igual manera, es posible concluir que esta formación, y los aprendizajes obtenidos en ella, impactan positivamente el proceso de enseñanza-aprendizaje. Sin embargo, a pesar de los múltiples beneficios y aplicaciones, se reconoce la necesidad de abordar las diversas limitaciones reconocidas para que su aplicación sea aún más efectiva. 


\section{REFERENCIAS}

Anderson, S. (2010). Liderazgo directivo: claves para una mejor escuela. Psicoperspectivas, 9, 34-52. Baquero Barato, J., \& Rodríguez Moneo, M. (2016). La relación entre el proceso de autorregulación y el proceso de coaching. Universitas Psychologica, $15(1), \quad 141 \quad-152$. https://doi.org/10.11144/Javeriana.upsy15-1.rpap

Baquero Barato, J., \& Rodríguez Moneo, M. (2021). Can coaching contribute to the development of selfregulation? Similarities and differences between these two processes. Coaching: an international journal of theory, research and practice, https://doi.org/10.1080/17521882.2021.1929362

Bou, J. (2007). Coaching para docentes: el desarrollo de habilidades en el aula. Club Universitario. Carril, J. (2008). Zen coaching: un nuevo método que funde la cultura oriental y occidental para potenciar al máximo tu vida profesional y personal. Díaz de Santos.

Cortés, R. J. (2012). Coaching en el desarrollo profesional docente. Fases formativas y procesos metodológicos de investigación. Revista Electrónica Interuniversitaria de Formación del Profesorado, 15(4), 101-112.

Gornall, S., \& Burn, M. (2013). Coaching and learning in schools: a practical guide. SAGE.

Hall, M., \& Duval, M. (2010). Meta coaching. Trillas.

Hoekstra, A., \& Korthagen, F. (2011). Teacher Learning in a Context of Educational Change: Informal Learning Versus Systematically Supported. Journal of Teacher Education, 62 (1) 76-92.

Mitchel, J., \& Gansemer-Topf, A. (2016). Academic coaching and self-regulation: promoting the success of students with disabilities. Journal of Postsecondary Education and Disability, 29 (3), 249 - 256.

Pascual, R., \& Leonor, P. (2016). Impacto del Coaching Grupal sobre el Desarrollo de la Competencia de Autorregulación del Aprendizaje en Estudiantes de Ingeniería. Tesis de Maestría, Tecnológico de Monterrey.

Obiols, M., \& Giner, A. (2011). El modelo educativo de Bolonia y competencias docentes. Aportaciones desde el coaching educativo. Univest. Recuperado de: http://dugidoc.udg.edu/birstream/handle/10256/3726/321.pdf?sequence $=1$

Rogers, J. (2012). Coaching Skills: A Handbook. Maidenhead: McGraw-Hill Education.

Rose, J., McGuire-Snieckus, \& Gilbert, L. (2015). Emotion coaching - a strategy for promoting behavioural self-regulation in children/young people in schools: a pilot study. The European Journal of Social and Behavioural Sciences, XIII, 1766 - 1790.

Rosinski, P. (2008). Coaching y cultura. Gran Aldea Editores.

Rhodes, C., \& Fletcher, S. (2012). Coaching and mentoring for self-efficacious leadership in schools. International Journal of Mentoring and Coaching in Education, 2, 2 - 6.

Sánchez-Teruel, D. (2013). El coaching pedagógico dentro del sistema educativo: innovando procesos. Revista Intercontinental de Psicología y Educación, Julio-Diciembre, 171-191. 
Sánchez, B., \& Boronat, J. (2014). Coaching educativo: modelo para el desarrollo de competencias intra e interpersonales. Educación XXI, 17 (1), 219 - 242.

Van Nieuwerburgh, C. (2012). Coaching in Education: Getting Better Results for Students, Educators, and Parents. Karnac Books.

Villa, J., Caperán, J., \& Caperán, J. (2010). Manual de coaching: cómo mejorar el rendimiento de las personas. Profit Editorial.

Whitmore, J. (2003). Coaching: el método para mejorar el rendimiento de las personas. Barcelona: Paidós. 